\title{
Comparative Analysis of Human Motion Trajectory Prediction using Minimum Variance Curvature
}

\author{
Gonzalo Ferrer \\ Institut de Robòtica i Informàtica Industrial \\ Llorens Artigas 4-6 \\ Barcelona, Spain \\ gferrer@iri.upc.edu
}

\author{
Alberto Sanfeliu ${ }^{*}$ \\ Institut de Robòtica i Informàtica Industrial \\ Llorens Artigas 4-6 \\ Barcelona, Spain \\ sanfeliu@iri.upc.edu
}

\begin{abstract}
The prediction of human motion intentionality is a key issue towards intelligent human robot interaction and robot navigation. In this work we present a comparative study of several prediction functions that are based on the minimum curvature variance from the current position to all the potential destination points, that means, the points that are relevant for people motion intentionality. The proposed predictor computes, at each interval of time, the trajectory from the present to the destination positions, and makes a prediction of the human motion at each interval of time using only the criterion of minimum curvature variation. The method has been validated in the Edinburgh Informatics Forum Pedestrian database.
\end{abstract}

\section{Categories and Subject Descriptors}

H.1.2 [Models and Principles]: User/Machine SystemsHuman information processing

\section{General Terms}

Algorithms

\section{HUMAN MOTION PREDICTION}

The design and development of intelligent social mobile robots that interact with humans in daily living activities requires the design of new tools that allow the understanding of human motion intentionality. A prior knowledge of human motion intentionality would allow to optimize robot movements, optimize robot trajectories and find out the most adequate human-robot motion behaviors.

Human motion patterns have been studied widely. Of special interest is the paper of Arechavaleta et al. [1] where they analyze human walking from the perspective of finding an optimal principle for human locomotor trajectories in goal-directed walking. As a conclusion, a control law that minimizes curvature variation along the path is sought, resulting in a composition of arcs of clothoids whose curvature grows linearly with the distance from the origin.

\footnotetext{
${ }^{*}$ This research was conducted at the Institut de Robòtica i Informàtica Industrial (CSIC-UPC). It was partially supported by CICYT projects DPI2007-61452 and Ingenio Consolider CSD2007-018
}

Copyright is held by the author/owner(s).

HRI'11, March 6-9, 2011, Lausanne, Switzerland.

ACM 978-1-4503-0561-7/11/03.
The study of human motion patterns may be divided in two major categories of methods. The first set of methods is more related to a classification problem, like [2] and [5] where a primitive trajectory is built in order to predict. The second set of methods, [3] for instance, propose a human motion prediction based on goal-oriented intentionality.

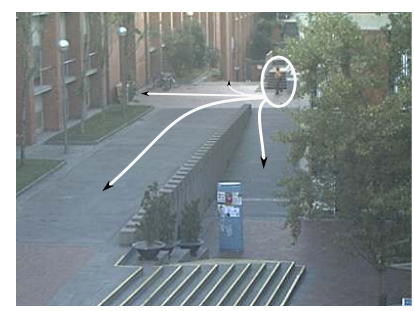

Figure 1: Example of human motion prediction, a step towards human-robot intelligent interaction.

The proposed scenario is a $2 \mathrm{D}$ free space in which a set of destination points are defined. In this paper, we will refer to destination points $D=\left\{D_{1}, D_{2}, \ldots, D_{N}\right\}$ to all those specific emplacements on an environment that are typically starting or finishing points for people trajectories. Throughout the paper we will assume the following hypothesis: people always move from an origin point to a destination point, so their walk is goal-directed.

Any given path, from $D_{i}$ to $D_{j}$ will be defined as a succession of poses $q_{i j}^{t}=\left[x_{i j}^{t}, y_{i j}^{t}, \theta_{i j}^{t}\right]$ defined at each time step $t \in[0, T]: S_{i j}=\left\{q_{i j}^{1}, q_{i j}^{2}, \ldots, q_{i j}^{T}\right\}$ wherein a distinction must be done between past trajectory $S_{i j}^{0: t}$ and future trajectory $S_{i j}^{t+1: T}$, or predicted trajectory. Human trajectory model is obtained from minimizing the curvature variance along a given path, from a starting point, usually the current position to the final destination. For the analysis, the following curvature definition is used: $\kappa=\frac{\partial \theta}{\partial s} \approx \frac{\Delta \theta}{\Delta s}$. This estimation is repeated for every final destination generating $N$ different trajectories $S_{i j}^{0: T}$ for every position analyzed.

$$
J_{i j}^{t}=\sum_{\tau=t}^{T}\left(\frac{\kappa_{i j}^{\tau}-\kappa_{i j}^{\tau-1}}{\triangle t}\right)^{2}
$$

Our approach solves the curvature minimization taking advance of geometric reasons, solving the boundary value problem from the current pose $q_{i j}^{t}$ to $D_{j}$ using the fourth degree spline that minimizes $J_{i j}^{t}$. The initial solution proposed by [1] is a constrained nonlinear optimization, which is indeed costly to solve. Of course one may think if a too coarse 
approximation invalidates the model; but a comparison between both techniques suggests the opposite. Thereby the simplified trajectory model is enormously faster than other routines besides much easier to implement. We would like to underline the previous statement, since it may be considered as the main strength of the present work.

Once generated all possible $M$ paths to its corresponding destinations, a decision function is needed in order to predict future trajectories. The minimum curvature parameter $J_{i j}^{t}$ is used in all the prediction functions proposed: the first one takes into account only the curvature variance, the curvature predictor $C P$ finds the minimum $J_{i j}^{t}$; the second one takes into account both the length and the curvature variance, $C L P$ selects the maximum $F_{i j}^{t}$; and the third one weights an average of previous predictions in a limited time window, $W C L P$ looks for a maximum too.

$$
\begin{gathered}
\mathrm{CLP}: F_{i j}^{t}\left\{J_{i j}^{t}, L_{i j}^{t}\right\}=\frac{1 / L_{i j}^{t} J_{i j}^{t}}{\sum_{n=1}^{N} 1 / L_{i n}^{t} J_{i n}^{t}} \\
\text { WCLP : } W F_{i j}^{t}=\sum_{w=0}^{W} \alpha_{w} F_{i j}^{t-w}
\end{gathered}
$$

\section{RESULTS AND CONCLUSIONS}

We have used the Edinburgh Informatics Forum Pedestrian database [4] consisting of a set of detected targets of people walking through the Informatics Forum, the main building of the School of Informatics at the University of Edinburgh. For our validation test, we have used a randomly selected subset of the database, 100 trajectories for comparing the results of the different curvature variance predictors at each point of the path walked by the target so far. We have evaluated the three different predictors: $C P, C L P$ and $W C L P$.

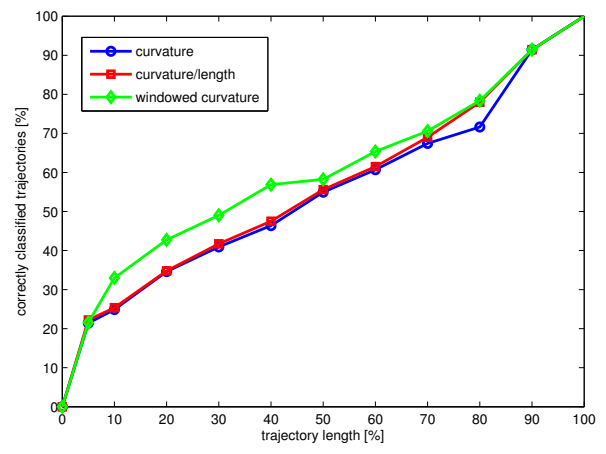

Figure 2: Correctly classification of 100 trajectories through the different curvature variance methods.

In the database of trajectories of the human motions, we have seen that there are people walking from an origin to a destination without stopping, maintaining a constant speed while other people stop and modify their trajectory several times before reaching their destination. All these cases have been also evaluated in the validation test.

Fig. 2 corresponds to the results of the three proposed predictors. An evaluation of the prediction accuracy related to the length of the trajectory observed is plotted. Intuitively, the more we observe a trajectory, the more accurate a prediction becomes. The $W C L P$ performs slightly better on early stages of prediction, but at the end, when a destination is clearly envisaged, all predictors success.

The method has been validated using the Edinburgh Informatics Forum real-world database of human walking in a public scenario. The results show that our predictor functions works well for these scenarios and surprisingly what is more important is that the predictor showed an acceptable performance when evaluating complex trajectories, such as destination changes, unexpected behaviors and other abnormalities, that typical methods might fail to identify while the curvature criterion rapidly recognizes the new intentionality. Specially interesting is the hypothesis of human navigation strategy as a consequence of goal achieving of consecutive destinations.

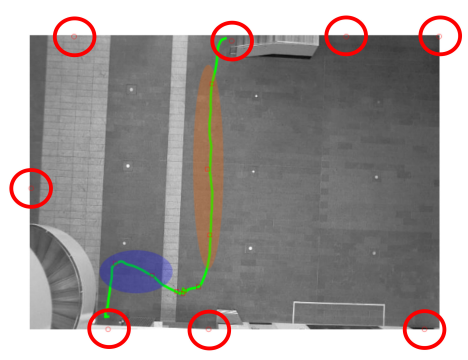

Figure 3: Study of a challenging trajectory. The initial part (blue area) clearly entails an implicit intentionality towards destination $D_{6}$. Before arriving, the subject stops and then changes its intentionality (orange area) to the destination $D_{2}$.

As a conclusion, we have proposed a method for evaluating, at every single point, the prediction function associated to every final destination. We have defined three different predictors and the best one has been the time window predictor. Although this approach outperformed the CP and CLP estimators, a more complete scheme, ideally a probabilistic framework, could enhance prediction accuracy. Unfortunately, this problem was out of the scope of the present paper, remaining as an opened issue for future work.

\section{REFERENCES}

[1] G. Arechavaleta, J.-P. Laumond, H. Hicheur, and A. Berthoz. An optimality principle governing human walking. IEEE Transactions on Robotics, 24(1):5, Feb. 2008.

[2] M. Bennewitz, W. Burgard, G. Cielniak, and S. Thrun. Learning motion patterns of people for compliant robot motion. The International Journal of Robotics Research, 24(1):31, 2005.

[3] A. Foka and P. Trahanias. Probabilistic Autonomous Robot Navigation in Dynamic Environments with Human Motion Prediction. International Journal of Social Robotics, 2(1):79-94, 2010.

[4] B. Majecka. Statistical Models of Pedestrian Behaviour in the Forum. Msc dissertation, School of Informatics, University of Edinburgh, 2009.

[5] D. Vasquez, T. Fraichard, and C. Laugier. Markov Models : An Incremental Tool for Learning and Predicting Human and Vehicle. The International Journal of Robotics Research, 28(11):1486-1506, 2009. 